\title{
IDENTIFICATION OF ACETATES IN Elasmopalpulus lignosellus PHEROMONE GLANDS USING A NEWLY CREATED MASS SPECTRAL DATABASE AND KÓVATS RETENTION INDICES
}

\author{
Gulab N. Jham* and Alexsandro A. da Silva \\ Departamento de Química, Universidade Federal de Viçosa, 36571-000 Viçosa - MG, Brasil \\ Eraldo R. Lima \\ Departamento de Biologia Animal, Universidade Federal de Viçosa, Viçosa - MG, Brasil \\ Paulo A. Viana \\ Embrapa-Milho e Sorgo, Sete Lagoas - MG, Brasil
}

Recebido em 7/6/06; aceito em 6/9/06; publicado na web em 26/3/07

\begin{abstract}
Based on a specially created mass spectral database utilizing 23 tetradecenyl and 22 hexadecenyl acetate standards along with Kóvats retention indices obtained on a very polar stationary phase [poly (biscyanopropyl siloxane)] (SP 2340), (Z)-9-hexadecenyl acetate, (Z)-11-hexadecenyl acetate and (E)-8-hexadecenyl acetate were identified in active pheromone extracts of Elasmopalpus lignosellus. This identification was more efficient than our previous study using gas chromatography/mass spectrometry with a dimethyl disulfide derivative where we could only identify the first two acetates. The acetate composition of the pheromone gland differed from region to region in Brazil and from that from the Tifton (GA, USA) population, suggesting polymorphism or a different sub-species.
\end{abstract}

Keywords: Elasmopalpus lignosellus; acetates; polymorphism.

\section{INTRODUCTION}

Elasmopalpus lignosellus is a major pest that attacks corn in Brazil and granaries worldwide ${ }^{1,2}$, controlled by extensive use of toxic insecticides. Ecologically accepted methods for its control are highly desirable and the identification of a sex pheromone in $E$. lignosellus $^{3}$ opened such a possibility. Ten acetates and alcohols were identified by gas chromatography-mass spectrometry (GCMS) in the pheromone gland of E. lignosellus from USA population in Georgia. The composition of the pheromone mixture was determined through field tests ${ }^{4}$. However, the commercially available sex pheromone blend in the USA produced a very low response when evaluated in field experiments in Brazil ${ }^{5}$. This led to the hypothesis that the pheromone blend of E. lignosellus in Brazil might be different due to regional differences. This hypothesis was confirmed since the acetate composition and activity of the pheromone extract varied from region to region in Brazil as well as from that in the USA ${ }^{6}$. However, in that study, it was not possible to identify the three acetates in the active extracts the gland extract obtained from insects in the state of Minas Gerais (extract 1). We could identify only (Z)-9-hexadecenyl acetate (Z)-9-HDA and (Z)11-hexadecenyl acetate $(Z)$-11-HDA and confirm their presence by DMDS derivative ${ }^{6}$. The third acetate, either $(E)-8$-hexadecenyl acetate [(E)-8-HDA] or (E)-9-hexadecenyl acetate [(E)-9-HDA] could not be identified based on its mass spectra or by comparison of the retention time $\left(t_{R}\right)$ with standards. In addition, the DMDS ${ }^{7}$ derivative, which frequently identifies the double bond position in mono unsaturated acetates, could not be detected when the pheromone extract was reacted with $\mathrm{CH}_{3}-\mathrm{S}-\mathrm{S}-\mathrm{CH}_{3} / \mathrm{I}_{2}$. In this paper we report the identification of the three acetates in extract 1 using a newly created mass spectral database and Kóvats retention index (KI). In addition, this approach was used to identify acetates in another pheromone E. lignosellus gland extract prepared from an insect colony obtained from the state of Goiás (extract 2).

*e-mail: gulab@ufv.br

\section{EXPERIMENTAL}

\section{GC-MS analysis}

All the 23 possible isomers of tetradecenyl acetates (TDAs) and 22 out of the 27 possible hexadecenyl acetates (HDAs) [(Z) and $(E)$ isomers of $\Delta^{2}$ to $\Delta^{13}$ ] (99\% pure) were obtained from the Research Institute for Plant Protection (Wageningen, The Netherlands). Data were obtained on a two instruments. The data to calculate Kováts retention indices (KIs), were obtained on a gas chromatograph (Shimadzu, Kyoto, Japan, model GC 17 A) fitted with a FID, an auto sampler and workstation. Mass spectral data were obtained on a gas chromatograph-mass spectrometer (Shimadzu, Kyoto, Japan, model QP 5000 and software programClasss-5000, Version.1.2) fitted with an auto sampler, workstation and a database (Wiley 229) with about 350,000 (three hundred and fifty thousand) entries. Fused capillary columns (30 m x $0.25 \mathrm{~mm}$; film thickness of $0.25 \mu \mathrm{m}$ ) coated with the very polar stationary phases [poly (biscyanopropyl siloxane)] (SP 2340) and [poly(ethyleneglycol)] (Supelcowax) were purchased from Supeloco (Bellefonte, PA, USA) and [poly(dimethylsiloxane)] (apolar) (DB1) from J\&W Scientific (Folsom, CA, USA). In all cases, the GC oven temperature was programmed from 60 to $250{ }^{\circ} \mathrm{C}$ at a rate of 6 ${ }^{\circ} \mathrm{C} / \mathrm{min}$. One microliter of each standard mixture containing $5 \mathrm{ng}$ of each constituent was injected five times by the splitless with the split vent being closed for $30 \mathrm{~s}$. Helium (purity 99.995\%) was the carrier gas for all analyses at a flow rate of $1 \mathrm{~mL} / \mathrm{min}$ at a column pressure of $80 \mathrm{kPa}$. Electron ionization mass spectra $(70 \mathrm{eV})$ were recorded by scanning from $\mathrm{m} / \mathrm{z}, 29$ to 320 .

For determination of the KIs, a mixture of linear saturated hydrocarbons was added to each unsaturated acetate and the mixture analysed by gas chromatography. KIs were calculated in a worksheet from the following formula: $\mathrm{KI}=100 \mathrm{y}+100(\mathrm{z}-\mathrm{y}) \mathrm{X}_{\mathrm{R}(\mathrm{x})}-\mathrm{t}_{\mathrm{R}(\mathrm{y})} / \mathrm{t}_{\mathrm{R}(\mathrm{z})}$ $t_{R(y)}$, where $y$ and $z$ are the carbon numbers of the hydrocarbons eluting before and after a given acetate, respectively; $t_{R(x)}$ is the retention time of the acetate; $t_{\mathrm{R}(\mathrm{y})}$ and $\mathrm{t}_{\mathrm{R}(\mathrm{z})}$ are the retention times of the 
hydrocarbons eluting before and after the acetate, respectively. KI for each mixture was determined five times (one for each injection).

For each of the five GC-MS runs, the background-subtracted spectra were recorded. To obtain representative data, the mass spectra over the entire GC peaks ( $\sim 50$ scans) of interest were grouped and subtracted from the grouped mass spectra of the region closest (before or after) to where no compounds eluted ( $\sim 50$ scans). From the five values thus obtained, the averages and standard deviations (SD) of the major ions were computer-calculated using the Microsoft Excel program. All the mass spectra were stored as five replicate entries in a reference library (PheroAcetates).

\section{Preparation and evaluation of pheromone gland extracts}

The two pheromone extracts prepared in our previous study were used for this study ${ }^{6}$. Briefly, two Elasmopalpus lignosellus colonies were reared in our laboratory, the first being initiated from pupae obtained from a cornfield in the region of Sete Lagoas, Minas Gerais and the second from a sugarcane field in the region of Goiânia, Goiás. Both colonies were maintained in an artificial diet.

From the two colonies, two extracts were prepared from the pheromone glands of virgin E. lignosellus females. The extract obtained from the first colony was designated as extract 1 while the extract obtained from the second colony was designated as extract 2. During the calling period, the pheromone gland of 1-2day-old females was removed and extracted with $100 \mu \mathrm{L}$ of hexane for $2 \mathrm{~h}$. The extracts were concentrated to about $30 \mu \mathrm{L}$ by a gentle stream of $\mathrm{N}_{2}$ and $1 \mu \mathrm{L}$ was injected into the GC-MS. After GC-MS runs, the mass spectra of the major peaks were compared on line using PheroAcetates and the search algorithms provided by the Shimadzu GC-MS software program (Classs-5000, Version.1.2). After comparison, the data system provided a similarity index (SI) for each unknown compound. Only those with an SI index greater than $90 \%$ were considered as significant predictions. The mass spectra of peaks identified as acetates by PheroAcetates were examined to confirm their presence. After confirmation, the KIs of these peaks were determined by co-injecting appropriate hydrocarbons. This procedure was repeated five times.

\section{RESULTS AND DISCUSSION}

GC-MS and GC-EAD (gas chromatography-electroantennography) analysis of active E. lignosellus extracts along with electroantennography (EAG) with puffs on the males' antenna led to the identification of (Z)-9- HDA and (Z)-11-HDA in extract 1 , and (Z)-9-TDA and (Z)-11-HDA in extract $2^{6}$. The composition was very different from the one reported for the same insect in Tifton, Ga, USA where the following ten compounds were identified by GCMS $^{4}$ : (Z)-7-TDA (16.5\%), (Z)-9-TDA (8.8\%), tetradecyl acetate (3.8\%), (Z)-9-HDA (28.8\%) (Z)-11-HDA (7.4\%), hexadecyl acetate (9.6\%), (Z)-7-tetradecenol (2.1\%), (Z)-9-tetradecenol (7\%) and (Z)11-hexadecenol (14\%). The activity and chemical compositions let us to conclude polymorphism in E. lignosellus in Brazil as well as in USA $^{6}$. Also, the possibility of the existence of sub-species cannot be ruled out. In moths, the relative proportions of sex pheromone components and the specificity of the male behavioural response are often decisive for reproductive isolation between closely related species that overlap in time and space ${ }^{8-10}$. Significant inter-population variation in pheromone blends and male response has been demonstrated in several moth species ${ }^{11-13}$. It appears this is the case with E. lignosellus in relation to the population studied in the USA ${ }^{4}$. In another study, sex pheromone "dialects" have been reported in Agrotis segetum from different geographic regions ${ }^{14}$.
However, in extract 1 we could identify only two of the three acetates in the pheromone gland extract of E. lignosellus acetates thorough the conventional GC-MS and DMDS derivative. The third acetate could either be (Z)-8-HDA or (E)-8-HDA. Reaction of the

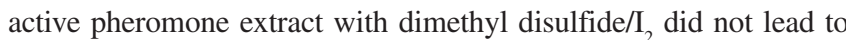
the detection of the DMDS derivatives of $(E)-8$ or $(E)-9-\mathrm{HDA}$. We faced a similar problem with the identification of pheromone of Tuta absoluta ${ }^{15}$. In that study, we were unable to prepare the DMDS derivative of (E)-3-TDA but (Z)-8-TDA and (Z)-11-TDA presented no problems. In this study as well as in the Tuta absoluta study, it was possible to form the DMDS derivatives with standard compounds on a nanogram scale.

To identify the third acetate in extract 1 , we attempted to conduct identification based mass spectral database commonly used to identify essential oils ${ }^{16}$. However, commercially available mass spectral databases such as Wiley or NIST contain very limited data on pheromone-like acetates. To overcome this limitation, we also created a specialized mass spectral database using 45 acetates (23 TDAs and 22 HDAs) and named it PheroAcetates. The database was used along with KIs of 43 acetates determined on a capillary column coated with very polar stationary phase [poly (biscyanopropyl siloxane)]. Although the KIs of all TDAs and HDAs isomers (except the $\Delta^{2}$ ) were reported on DB-5 (non polar) and Carbowax coated capillary columns ${ }^{17}$, they could not be used in this study due to inadequate resolution with E. lignosellus pheromone extracts on these columns (Figure 1). This result was in agreement with our previous studies with other pheromone extracts $^{7}$ and literature reports ${ }^{18,19}$. Since a better resolution was on the very polar stationary phase [poly (biscyanopropyl siloxane)], we obtained KIs on this stationary phase.
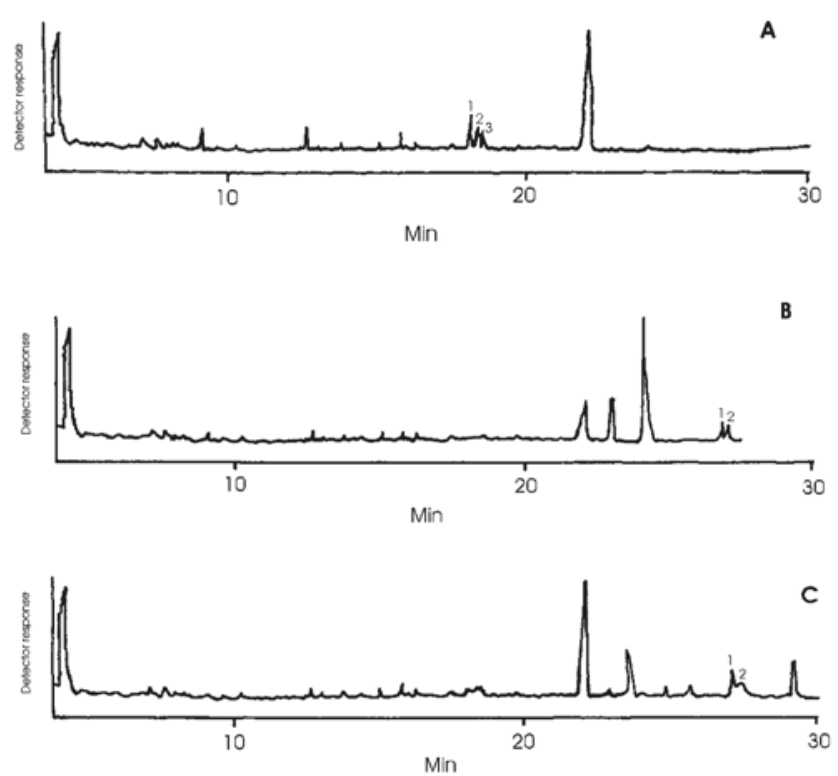

Figure 1. Sections of the reconstructed gas chromatograms obtained from the volatiles in the pheromone extract of Elasmopalpus lignosellus using a fused silica column coated with the [poly (biscyanopropyl siloxane)] (A), [poly (dimethylsiloxane)] (B) and [poly (ethyleneglycol)] (C) phases. Peaks 1, 2 and 3 were identified as (E)-8-HDA, (Z)-9-HDA and (Z)-11-HDA, respectively (A). Peak 1 was identified as a mixture of (E)-8-HDA+(Z)-9HDA and peak 2 as (Z)-11-HDA ( $B$ and $C$ )

All the standards evaluated in this study presented one peak (>99\%) on GC-MS analysis. This was in agreement with the supplier's specification. It was important to confirm this data. Under the GC-MS conditions used, within a replicate set of five injections, excellent 
reproducibility of $t_{R}$ was obtained ( $\mathrm{SD}<0.007 \mathrm{~min}$ ) (data not shown). All the compounds eluted during the temperature programming. To calculate the KIs, an appropriate formula, which considers temperature programming was utilized ${ }^{20}$. KIs were highly reproducible with the variation between each run being about two units.

The relative ion intensities in the mass spectra of compounds obtained through GC-MS analysis is data processing dependent. This is specially true in trace analysis as with pheromone where amount of active compounds is usually small (ng or $\mathrm{pg}$ ). To obtain representative mass spectral data for PheroAcetates, we used $5 \mathrm{ng}$ of standard acetates. We took great care to obtain representative background subtracted mass spectra of standards. Average mass spectra were obtained by scanning the entire GC peak from which the background was subtracted. The region to subtract the background was carefully chosen. In the case of standards, this was easily possible as regions where no peaks eluted were available. Background subtracted mass spectra thus obtained were highly reproducible with the SD being less than $1 \%$ for the major ions. In the case of pheromone samples, the background subtraction was more difficult as not always, regions close to where peaks of interest were always clean. About 50 scans were used.

The mass spectra of all the standards were typical of linear long chain acetates with the base peak at $m / z, 43\left(\mathrm{CH}_{3} \mathrm{CO}^{+}\right)$, and moderately intense ( $6 \%$ ), corresponding to $\left.\mathrm{M}^{+}-\mathrm{CH}_{3} \mathrm{COOH}\right)$. No molecular ions were recorded. In addition, the mass spectra were characterized by ions at intervals of 14 units. In these clusters $(\mathrm{m} / z 50$ to 140), ions corresponding to $\left(\mathrm{C}_{\mathrm{n}} \mathrm{H}_{2 \mathrm{n}-1}\right)^{+}$and $\left(\mathrm{C}_{\mathrm{n}} \mathrm{H}_{2 \mathrm{n}}\right)^{+}$were more intense than the $\left(\mathrm{C}_{\mathrm{n}} \mathrm{H}_{2 \mathrm{n}+1}\right)^{+}$. Although the relative peak intensities in each spectrum were unique to each compound, the basic mass spectra were the same with differences in the relative intensities of the cluster ions in the region between $\mathrm{m} / \mathrm{z}, 50$ to 140 . We realized that the similarities in the mass spectra of the isomers within a series would make online identification difficult by the database. Ions corresponding to (M$\left.\mathrm{CH}_{3} \mathrm{COOH}\right)^{+}$aided detection of TDAs and HDAs in insect extracts.

Figure 1A presents a reconstructed gas chromatogram obtained on GC-MS analysis of pheromone extract of E. lignosellus with [poly (biscyanopropyl siloxane)] stationary phase. Peaks 1, 2 and 3 were identified as HDAs by PheroAcetates (Table 1). Careful scanning suggested that these peaks were homogenous as no significant variations in the mass spectra occurred. Mass spectral examination of all the peaks in the reconstructed gas chromatogram confirmed that only three HDAs were present (Figure 2). As can be observed the mass spectra were very similar. However, PheroAcetates predicted 3-4 isomers for each peak. This is common with mass spectral data prediction and would be specially isomers given the similarities in the mass spectrum. Apparently, the matching algorithm of the software package used in this study for database search was not sophisticated enough to take maximum advantage of these peak intensity differences. Nevertheless, it should be pointed out that despite the existence of several peaks in the gas chromatogram, PheroAcetates carried out no misidentification of acetates.
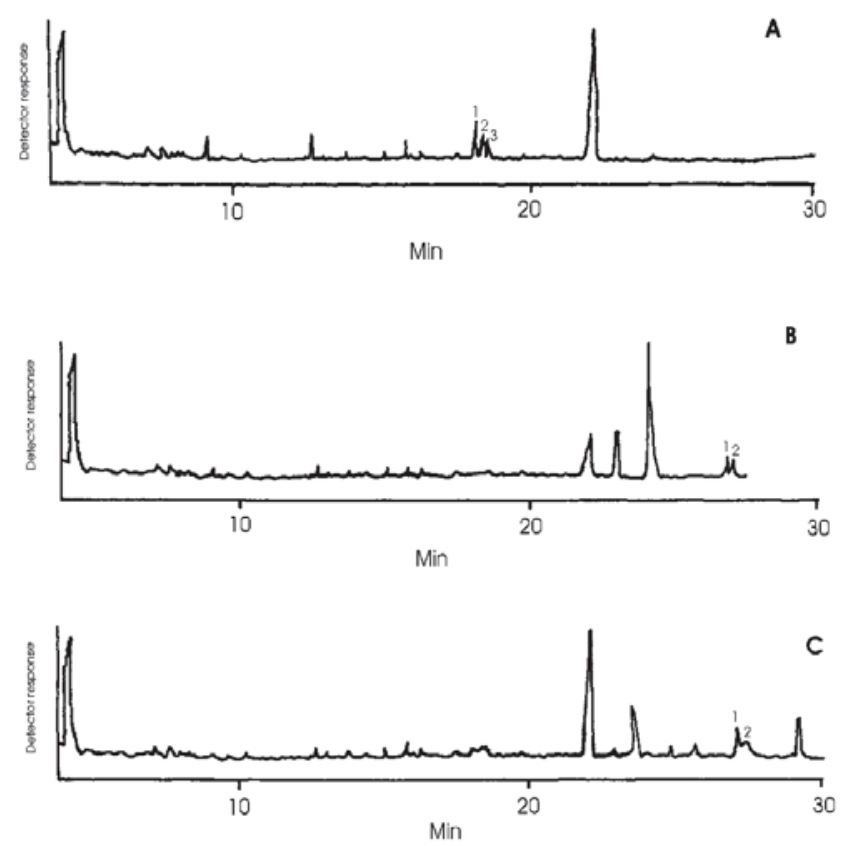

Figure 2. Mass spectrum of peak $1(A)$, peak $2(B)$ and peak $3(C)$ obtained from the pheromone extract of Elasmopalpus lignosellus using a fused silica column coated with the [poly (biscyanopropyl siloxane)] stationary phase (Figure 1A), corresponding to (E)-8-HDA, (Z)-9-HDA and (Z)-11-HDA, respectively

The number of possible isomers for the three peaks in the pheromone extract 1 was reduced by KI determination. The KIs were very reproducible in this study with the variation being about two KI units with standards and pheromone extracts (five repetitions). Thus using $\pm 2 \mathrm{KI}$ as the basis for comparison, peaks 1 and 3 were identified as (E)-9-HDA and (Z)-11-HDA, respectively while peak 2 could be (Z)-9-HDA or $(E)$-13-HDA. All these compounds were predicted by PheroAcetates. The final identity of peak 2 was established to be (Z)-9-HDA based on the combination of KI and PheroAcetates prediction. Hence, peaks 1, 2 and 3 were identified as (E)-8-HDA, (Z)-9-HDA and (Z)-13-HDA, respectively. In the pheromone extract 2 , the two acetates, $(Z)-11$-HDA and $(Z)$ 9-TDA were confirmed. In addition, using the same approach we confirmed the presence of (Z)-9-TDA and (Z)-11-HDA in another gland extract obtained from Goiás (extract 2).

\section{CONCLUSIONS}

Based on PheroAcetate/KI we were able to unambiguously determine the acetate composition of pheromone gland of $E$. lignosellus. This was not possible with the conventional approach

Table1. Identification of hexadecenyl acetates (HDAs) in the pheromone gland of Elasmopalpulus lignosellus (extract 1) based database and Kováts indices obtained on the fused silica capillary column coated with the highly polar stationary phase [poly (biscyanopropyl siloxane)]

\begin{tabular}{|c|c|c|c|c|}
\hline aPeak no. & $\begin{array}{l}\text { HDAs predicted by the } \\
\text { PheroAcetates mass } \\
\text { spectral database [SI 90\%] }\end{array}$ & $\begin{array}{l}\text { Average KI of the } \\
\text { peak in the } \\
\text { pheromone extract }\end{array}$ & $\begin{array}{l}\text { HDAs identified in } \\
\text { the pheromone extract } \\
\text { based on } \pm 2 \mathrm{KI}\end{array}$ & $\begin{array}{l}\text { HDAs identified in the } \\
\text { pheromone extract based on } \\
\pm 2 \mathrm{KI} \text { and PheroAcetates }\end{array}$ \\
\hline 1 & (Z)-7-HDA, $(E)$-8-HDA and (Z)-13-HDA & $2372^{\mathrm{b}}$ & (E)-8-HDA & (E)-8-HDA \\
\hline 2 & (Z)-9-HDA, $(E)-11$-HDA and $(E)-7-\mathrm{HDA}$ & $2388^{\mathrm{c}}$ & (Z)-9-HDA or $(E)-13$-HDA & (Z)-9-HDA \\
\hline 3 & (Z)-11-HDA, (Z)-13-HDA and (E)-13-HDA & $2402^{d}$ & (Z)-11-HDA & (Z)-11-HDA \\
\hline
\end{tabular}

a: Numbers refer to chromatographic peaks labelled in Figure 1A; b: average of 2372, 2371, 2372, 2371 and 2373; c: average of 2388, 2387, 2388, 2389 and 2388; d: average of 2401, 2401, 2402, 2402 and 2402. 
using mass spectra, $t_{\mathrm{R}}$ and DMDS derivative formation. The mass spectral database and KI could be successfully used to identify mono unsaturated acetates in lepidopteran pheromones.

\section{ACKNOWLEDGEMENTS}

We thank the Brazilian Government (CNPq, FINEP and FAPEMIG) for financial support. Scholarship from CNPq (G. N. Jham) is also gratefully accepted.

\section{REFERENCES}

1. Funderburk, J. E.; Herzog, D. C.; Lynch, R. E.; J. Entomol. Sci. 1987, 22, 159

2. Viana, P. A.; $2^{\circ}$ Simpósio Latino Americano de cana-de-açúcar, Maceió, Brasil, 1993.

3. Payne, T. L.; Smith; J. H.; Environ. Entomol. 1975, 4, 355

4. Lynch, R. E.; Klun, J. A.; Leonhardt, B. A.; Schwart, M.; Garner J. W.; Environ. Entomol. 1984, 13, 121.

5. Pires, C. S .S.; Vilela, E. F.; Viana, P. A.; Ferreira, J. T. B.; An. Soc. Ent. Bras. 1992, 21, 417.

6. Jham, G. N.; Silva, A. A.; Eraldo, R.; Viana, P.; J. Sep. Sci. 2005, 28, 281.

7. Francis, G. W.; Veland, K.; J. Chromatogr. 1981, 219, 379.
8. Sanders, C. J.; Science 1971, 171, 911.

9. Tumlinson, J. H.; Yonce, C. E.; Doolittle, R. E.; Heath, R. R.; Gentry, C. R.; Mitchell, E. R.; Science 1974, 185, 614

10. Cardé, R. T.; Cardé, A. M.; Hill, A .S.; Roelofs, W. L.; J. Chem. Ecol. 1977, 3,71 .

11. Löfstedt, C.; Löfqvist, J.; Lanne B. S.; van der Pers, J. N.; Hansson, C. B.; Oikos 1986, 46, 250.

12. Byers, J. R.; Struble, D. L.; J. Chem. Ecol. 1990, 16, 2981.

13. Byers, J. R.; Struble, D. L.; Herlne, C. E.; Kozub, G. C.; Lafontaine J. D.; J. Chem. Ecol. 1990, 16, 2969.

14. Hansson, B. S.; Löfstedt, C.; Szöcs, G.; Subchev, M.; Löfqvist. J.; J. Chem Ecol. 1990, 16, 1611 .

15. Attygalle, A. B.; Jham, G. N.; Svatoš, A.; Frighetto, R. T.; Meinwald, J.; Vilela, E. F.; Ferrara, F. A.; Bioorg. Med. Chem. 1996, 4, 305.

16. Adams, R. P.; Identification of Essential Oils by Gas chromatography/Mass spectrometry, Allured Publishing: Carol Stream, 1995.

17. Marques, F. A.; Mcelfresh, J. S.; Millar, J. G.; J. Braz. Chem. Soc. 2000, $11,592$.

18. Heath, R. R.; Tumlinson, J. H.; Hummel, H. E.; Miller, T. A.; Techniques in Pheromone Research, Springer Verlag: Berlin, 1984.

19. Hogge, L. R.; Millar, J. G.; Giddings, J. C.; Gruskha, E.; Brown, P. R.; Advances in Chromatography, Marcel Dekker: New York, 1987.

20. Robards, K.; Haddad, P. R.; Jackson, P. E.; Principles and Practices of Modern Chromatographic Methods, Academic Press: New York, 1994. 